\title{
The Use of Microwave Irradiation in Organic Synthesis. From Laboratory Curiosity to Standard Practice in Twenty Years
}

\author{
C. Oliver Kappe*
}

\begin{abstract}
In the past few years, the use of microwave energy to heat and drive chemical reactions has become increasingly popular in the medicinal chemistry community. First described 20 years ago, this non-classical heating method has matured from a laboratory curiosity to an established technique that is heavily used in academia and industry. One of the many advantages of using rapid 'microwave flash heating' for chemical synthesis is the dramatic reduction in reaction times: from days and hours to minutes and seconds. As will be discussed here, there are good reasons why many organic chemists are incorporating microwave chemistry into their daily work routine.
\end{abstract}

Keywords: Combinatorial chemistry · High-temperature chemistry · High-throughput synthesis . Microwave irradiation $\cdot$ Synthetic methods

\section{Introduction}

High-speed microwave synthesis has attracted a considerable amount of attention in recent years [1]. Since the first reports on the use of microwave heating to accelerate organic chemical transformations by the groups of Gedye and Giguere/Majetich twenty years ago [2], more than 3000 articles have been published in the area of microwave-assisted organic synthesis (MAOS). The initial slow uptake of the technology in the late 1980s and early 1990s has been attributed to its lack of controllability and reproducibility, coupled

\footnotetext{
*Correspondence: Prof. Dr. C.O. Kappe

Karl Franzens University Graz

Institute of Chemistry

Heinrichstrasse 28

A-8010 Graz

Tel.: +43 3163805352

Fax: + 433163809840

E-Mail: oliver.kappe@uni-graz.at
}

with a general lack of understanding of the basics of microwave dielectric heating. The risks associated with the flammability of organic solvents in a microwave field and the lack of available systems for adequate temperature and pressure controls were major concerns. Although most of the early pioneering experiments in MAOS were performed in domestic, sometimes modified, kitchen microwave ovens, the current trend clearly is to use dedicated instruments for chemical synthesis which have become available only in the last few years. Since the late 1990s the number of publications related to MAOS has therefore increased dramatically to a point where it might be assumed that in a few years most chemists will probably use microwave energy to heat chemical reactions on a laboratory scale. A survey indicates that the number of publications that report work utilizing dedicated microwave reactors is currently doubling every year (Fig. 1).

Not only is direct microwave heating able to reduce chemical reaction times from hours to minutes, but it is also known to reduce side reactions, increase yields and improve reproducibility. Therefore, many academic and industrial research groups are already using MAOS as a forefront technology for rapid reaction optimization, for the efficient synthesis of new chemical entities, or for discovering and probing new chemical reactivity. A large number of review articles [3] and several books [4] provide extensive coverage of the subject. The goal of the present short review is to highlight some of the basic facts and general trends in microwave synthesis without discussing individual chemistry examples.

\section{Microwave Theory}

Microwave irradiation is electromagnetic irradiation in the frequency range 0.3-300 GHz. All domestic 'kitchen' microwave ovens and all dedicated microwave reactors for chemical synthesis operate at a frequency of $2.45 \mathrm{GHz}$ (corresponding to a wavelength of $12.24 \mathrm{~cm}$ ) to avoid interference with telecommunication and cellular phone frequencies. The energy of the microwave photon at this frequency region $(0.0016 \mathrm{eV})$ is too low to break chemical bonds and is also lower than Brownian motion. It is therefore clear that microwaves cannot induce chemical reactions [5].

Microwave-enhanced chemistry is based on the efficient heating of materials by 'microwave dielectric heating' effects. Microwave dielectric heating is dependent on the ability of a specific material (i.e. a solvent or reagent) to absorb microwave energy and convert it into heat. The electric component of an electromagnetic field causes heating by two main mechanisms: dipolar polarization and ionic conduction. 


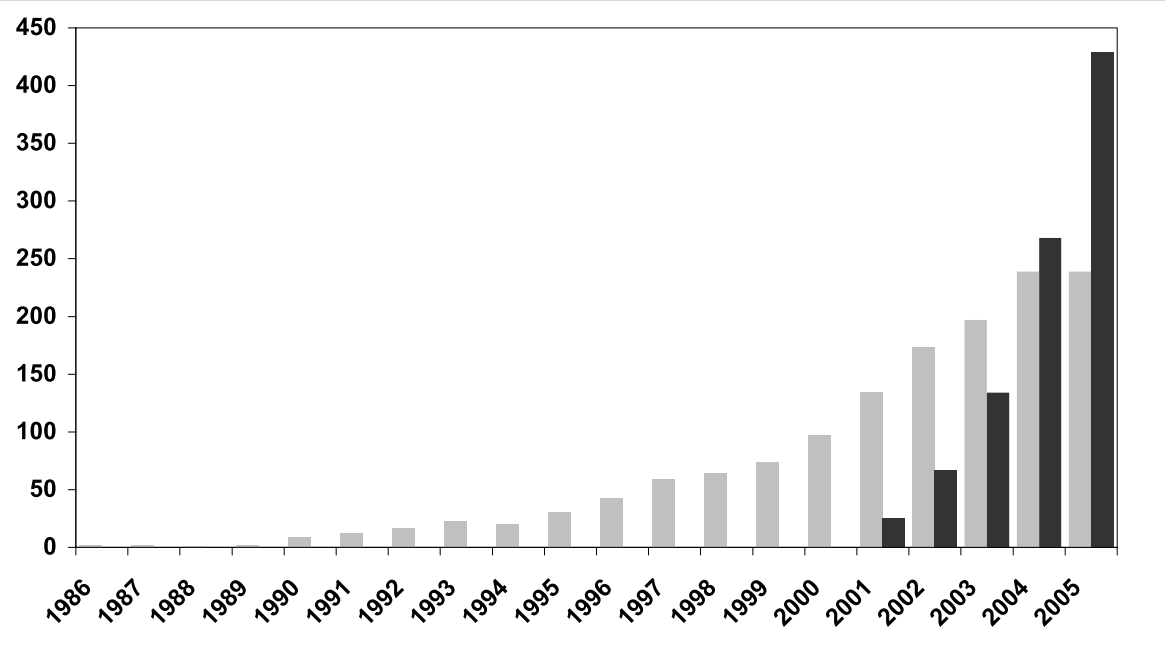

Fig. 1. Development in the number of publications on microwave-assisted organic synthesis. Shown in grey is the number of articles on MAOS published in seven synthetic organic chemistry journals from 1986-2005: Organic Letters, Journal of Organic Chemistry, Synthesis, Synlett, Tetrahedron Letters, Tetrahedron, Synthetic Communications (SciFinder Scholar keyword search on 'microwave'). Shown in black are the numbers of articles published in all major chemistry journals using MAOS under controlled conditions, i.e. using dedicated microwave reactors that allow temperature control (full text searches on 'microwave' in ca. 50 chemistry journals, only those articles dealing with synthetic organic chemistry were selected). Since most chemistry journals today do not encourage publication of microwave chemistry using domestic ovens, the total number of papers on MAOS is not increasing as strongly as the numbers of articles dealing with controlled MAOS.

When irradiated at microwave frequencies, the dipoles or ions of the sample align in the applied electric field. As the applied field oscillates, the dipole or ion field attempts to realign itself with the alternating electric field and, in the process, energy is lost in the form of heat through molecular friction and dielectric loss. The amount of heat generated by this process is directly related to the ability of the matrix to align itself with the frequency of the applied field. If the dipole does not have enough time to realign, or reorients too quickly with the applied field, no heating occurs. The allocated frequency of $2.45 \mathrm{GHz}$ used in all commercial systems lies between these two extremes and gives the molecular dipole time to align in the field, but not to follow the alternating field precisely [5].

The heating characteristics of a particular material (i.e. a solvent) under microwave irradiation conditions are dependent on the dielectric properties of the material. The ability of a specific substance to convert electromagnetic energy into heat at a given frequency and temperature is determined by the so-called loss tangent $\tan \delta$. The tangent loss factor is expressed as the quotient, $\tan \delta=\varepsilon " / \varepsilon$ ', where $\varepsilon$ " is the dielectric loss, indicative of the efficiency with which electromagnetic radiation is converted into heat, and $\varepsilon$ ' is the dielectric constant describing the ability of molecules to be polarized by the electric field. A reaction medium with a high $\tan \delta$ is required for efficient absorption and, consequently, for rapid heating. The loss tangents for some common organic solvents are summarized in the Table. In general, solvents can be classified as high $(\tan \delta>0.5)$, medium (tan $\delta$ 0.1-0.5), and low microwave absorbing $(\tan \delta<0.1)$. Other common solvents without a permanent dipole moment such as carbon tetrachloride, benzene, and dioxane are more or less microwave transparent. It has to be emphasized that a low tan $\delta$ value does not preclude a particular solvent from being used in a microwave-heated reaction. Since either the substrates or some of the

Table. Loss tangents $(\tan \delta)$ of different solvents $\left(2.45 \mathrm{GHz}, 20^{\circ} \mathrm{C}\right)^{\mathrm{a}}$

$\begin{array}{llll}\text { solvent } & \tan \delta & \text { solvent } & \tan \delta \\ \text { ethylene glycol } & 1.350 & \text { DMF } & 0.161 \\ \text { ethanol } & 0.941 & 1,2 \text {-dichloroethane } & 0.127 \\ \text { DMSO } & 0.825 & \text { water } & 0.123 \\ \text { 2-propanol } & 0.799 & \text { chlorobenzene } & 0.101 \\ \text { formic acid } & 0.722 & \text { chloroform } & 0.091 \\ \text { methanol } & 0.659 & \text { acetonitrile } & 0.062 \\ \text { nitrobenzene } & 0.589 & \text { ethyl acetate } & 0.059 \\ \text { 1-butanol } & 0.571 & \text { acetone } & 0.054 \\ \text { 2-butanol } & 0.447 & \text { tetrahydrofuran } & 0.047 \\ \text { 1,2-dichlorobenzene } & 0.280 & \text { dichloromethane } & 0.042 \\ \text { NMP } & 0.275 & \text { toluene } & 0.040 \\ \text { acetic acid } & 0.174 & \text { hexane } & 0.020\end{array}$

${ }^{a}$ data from ref. [4b] reagents/catalysts are likely to be polar, the overall dielectric properties of the reaction medium will in most cases allow sufficient heating by microwaves. Furthermore, polar additives such as $e . g$. ionic liquids or passive heating elements can be added to otherwise low absorbing reaction mixtures in order to increase the absorbance level of the medium.

Traditionally, organic synthesis is carried out by conductive heating with an external heat source (e.g. an oil-bath). This is a comparatively slow and inefficient method for transferring energy into the system since it depends on the thermal conductivity of the various materials that must be penetrated, and results in the temperature of the reaction vessel being higher than that of the reaction mixture. In contrast, microwave irradiation produces efficient internal heating (in core volumetric heating) by direct coupling of microwave energy with the molecules (e.g. solvents, reagents, catalysts) that are present in the reaction mixture. Since the reaction vessels employed are typically made out of (nearly) microwave transparent materials such as borosilicate glass, quartz or Teflon, an inverted temperature gradient as compared to conventional thermal heating results. The very efficient internal heat transfer results in minimized wall effects (no hot vessel surface) which may lead to the observation of so-called specific microwave effects, e.g. in the context of diminished catalyst deactivation.

\section{Microwave Effects}

Since the early days of microwave synthesis, the observed rate accelerations and sometimes altered product distributions 
compared to oil-bath experiments have led to speculation on the existence of so-called 'specific' or 'non-thermal' microwave effects [6]. Historically, such effects were claimed when the outcome of a synthesis performed under microwave conditions was different from the conventionally heated counterpart at the same apparent temperature. Reviewing the present literature it appears that today most scientists agree that in the majority of cases the reason for the observed rate enhancements is a purely thermal/kinetic effect, i.e. a consequence of the high reaction temperatures that can rapidly be attained when irradiating polar materials in a microwave field. As shown in Fig. 2, a high microwave absorbing solvent such as methanol ( $\tan \delta=0.659)$, can be rapidly superheated to temperatures $>100$ ${ }^{\circ} \mathrm{C}$ in excess of its boiling point when irradiated under sealed vessel microwave conditions. The rapid increase in temperature can be even more pronounced for media with extreme loss tangents such as ionic liquids where temperature jumps of $200{ }^{\circ} \mathrm{C}$ within a few seconds are not uncommon. Naturally, such temperature profiles are very difficult if not impossible to reproduce by standard thermal heating. Therefore, comparisons with conventionally heated processes are inherently troublesome.

Dramatic rate enhancements when comparing reactions that are performed at room temperature or under standard oilbath conditions (heating under reflux) with high-temperature microwave-heated processes have frequently been observed. As Mingos and Baghurst pointed out based on simply applying the Arrhenius law $(\mathrm{k}=\mathrm{A}$ $\left.\exp \left(-E_{\mathrm{a}} / \mathrm{R} T\right)\right)$, a transformation that requires 68 days to reach $90 \%$ conversion at $27{ }^{\circ} \mathrm{C}$ will show the same degree of conversion within 1.61 seconds (!) when performed at $227{ }^{\circ} \mathrm{C}[5]$. Due to the very rapid heating and extreme temperatures observable in microwave chemistry, it appears obvious that many of the reported rate enhancements can be rationalized by simple thermal/kinetic effects.

In addition to the above mentioned ther$\mathrm{mal} /$ kinetic effects, microwave effects that are caused by the uniqueness of the microwave dielectric heating mechanisms must also be considered. These effects should be termed 'specific microwave effects' and shall be defined as rate-accelerations that cannot be achieved or duplicated by conventional heating, but essentially are still thermal effects. In this category fall, for example (i) the superheating effect of solvents at atmospheric pressure, (ii) the selective heating of e.g. strongly microwave absorbing heterogeneous catalysts or reagents in a less polar reaction medium, (iii) the formation of 'molecular radiators' by direct coupling of microwave energy to specific reagents in homogeneous solu-

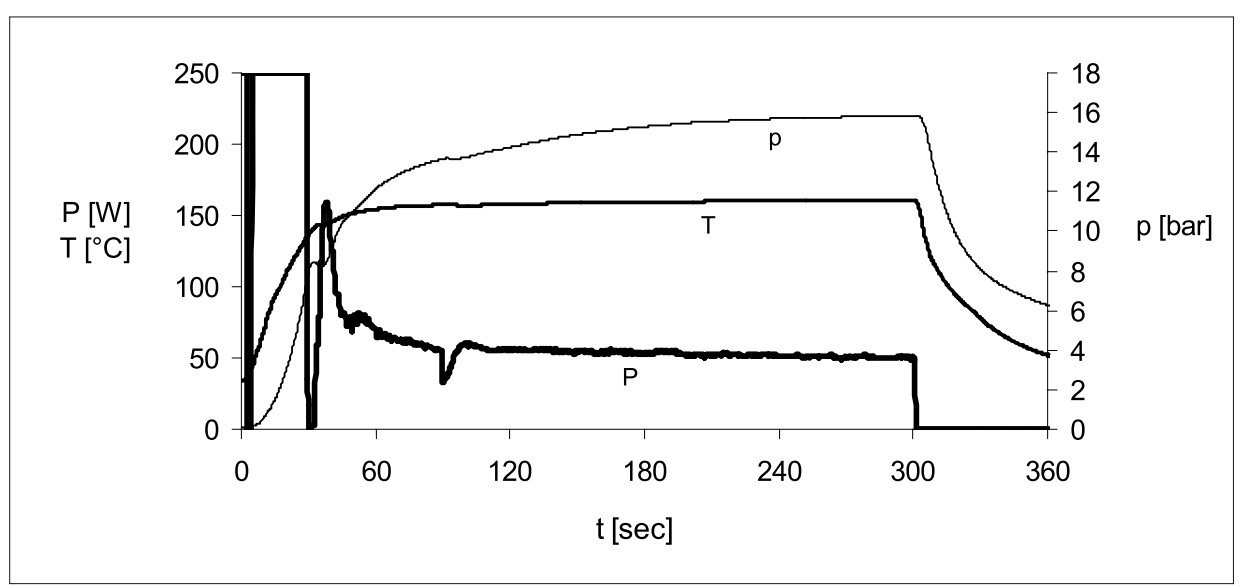

Fig. 2. Temperature $(T)$, pressure $(\mathrm{p})$, and power $(P)$ profile for a $3 \mathrm{ml}$ sample of methanol heated under sealed vessel microwave irradiation conditions. Single-mode microwave heating (250 W, 0-30 $\mathrm{sec})$, temperature control using the feedback from IR thermography (40-300 sec), and active gas-jet cooling (300-360 sec). The maximum pressure in the reaction vessel was ca. 16 bar. After the set temperature of $160{ }^{\circ} \mathrm{C}$ is reached, the power regulates itself down to ca. $50 \mathrm{~W}$.

tion (microscopic hotspots), and (iv) the elimination of wall effects caused by inverted temperature gradients. It should be emphasized that rate enhancements falling under this category are essentially still a result of a thermal effect (i.e. a change in temperature compared to heating by standard convection methods), although it may be difficult to experimentally determine the exact reaction temperature. In contrast, some authors have suggested the possibility of 'non-thermal microwave effects' (also referred to as athermal effects). These should be classified as rate accelerations that cannot be rationalized by either purely thermal/kinetic or specific microwave effects. Essentially, non-thermal effects result from a proposed direct interaction of the electric field with specific molecules in the reaction medium. It has been argued that the presence of an electric field leads to orientation effects of dipolar molecules and hence changes the pre-exponential factor $\mathrm{A}$ or the activation energy (entropy term) in the Arrhenius equation [6]. Furthermore, a similar effect should be observed for polar reaction mechanisms, where the polarity is increased going from the ground state to the transition state, resulting in an enhancement of reactivity by lowering of the activation energy [6]. Related to the issue of non-thermal or specific microwave effects is the recent concept that simultaneous external cooling of the reaction mixture (or maintaining subambient reaction temperatures) while heating by microwaves leads to an enhancement of the overall process (PowerMax, 'Enhanced Microwave Synthesis' [7]). Here, the reaction vessel is cooled from the outside by compressed air while being irradiated by microwaves. This allows a higher level of microwave power to be directly administered to the reaction mixture, but will prevent overheating by continuously removing latent heat. Micro- wave effects are the subject of considerable current debate and controversy and it is evident that extensive research efforts will be necessary in order to truly understand these and related phenomena.

\section{Processing Techniques}

Frequently used processing techniques employed in microwave-assisted organic synthesis involve solvent-less ('dry-media') procedures where the reagents are pre-adsorbed onto either a more or less microwave transparent (i.e. silica, alumina or clay) [8] or strongly absorbing (i.e. graphite) [9] inorganic support, that additionally can be doped with a catalyst or reagent. Particularly in the early days of MAOS the solvent-free approach was very popular since it allowed the safe use of domestic household microwave ovens and standard open vessel technology. While a large number of interesting transformations using 'dry-media' reactions have been published in the literature, technical difficulties relating to non-uniform heating, mixing, and the precise determination of the reaction temperature remain unsolved, in particular when scale-up issues need to be addressed. In addition, phase-transfer catalysis (PTC) has also been widely employed as a processing technique in MAOS [10].

Alternatively, microwave-assisted synthesis can be carried out using standard organic solvents either under open- or sealed vessel conditions [11]. If solvents are heated by microwave irradiation at atmospheric pressure in an open vessel, the boiling point of the solvent (as in an oil-bath experiment) typically limits the reaction temperature that can be achieved. In the absence of any specific- or non-thermal microwave effects the expected rate enhancements would be comparatively small. In order to nonethe- 
less achieve high reaction rates, high-boiling microwave absorbing solvents such as DMSO, NMP, 1,2-dichlorobenzene, or ethylene glycol (see the Table) have been frequently used in open-vessel microwave synthesis. However, the use of these solvents presents serious challenges during product isolation. Because of the recent availability of modern microwave reactors with on-line monitoring of both temperature and pressure, MAOS in sealed vessels has been celebrating a comeback in recent years. This is clearly evident surveying the recently published literature in the area of MAOS, and it appears that the combination of rapid dielectric heating by microwaves with sealed vessel technology (autoclaves) - a technique pioneered by Strauss [12] - will most likely be the method of choice for performing MAOS in the future.

\section{Equipment}

Although many of the early pioneering experiments in microwave-assisted organic synthesis have been carried out in domestic microwave ovens, the current trend undoubtedly is to use dedicated instruments for chemical synthesis. In a domestic microwave oven the irradiation power is generally controlled by on-off cycles of the magnetron (pulsed irradiation), and it is typically not possible to monitor the reaction temperature in a reliable way. Combined with the inhomogeneous field produced by the low-cost magnetrons and the lack of safety controls, the use of such equipment cannot be recommended. In contrast, all of today's commercially available dedicated microwave reactors for synthesis feature built-in magnetic stirrers, direct temperature control of the reaction mixture with the aid of fiber-optic probes or IR sensors, and software that enables on-line temperature/pressure control by regulation of microwave power output. Currently two different philosophies with respect to microwave reactor design are emerging: multimode and monomode (also referred to as single mode) reactors. In the so-called multimode instruments (conceptually similar to a domestic oven), the microwaves that enter the cavity are reflected by the walls and the load over the typically large cavity. In most instruments a mode stirrer ensures that the field distribution is as homogeneous as possible. In the much smaller monomode cavities, only one mode is present and the electromagnetic irradiation is directed through an accurately designed rectangular or circular wave guide onto the reaction vessel mounted in a fixed distance from the radiation source, creating a standing wave. The key difference between the two types of reactor systems is that whereas in multimode cavities several reaction vessels can be irradiated simultaneously in multi-vessel rotors (parallel synthesis), in monomode systems only one vessel can be irradiated at the time. In the latter case high throughput can be achieved by integrated robotics that move individual reaction vessels in and out of the microwave cavity. Most instrument companies offer a variety of diverse reactor platforms with different degrees of sophistication with respect to automation, database capabilities, safety features, temperature and pressure monitoring and vessel design (Fig. 3). Importantly, single-mode reactors processing comparatively small volumes also have a built in cooling feature that allows for rapid cooling of the reaction mixture by compressed air after completion of the irradiation period (see Fig. 2). The dedicated single-mode instruments available today can process volumes ranging from 0.2 to $\mathrm{ca}$. $50 \mathrm{ml}$ under sealed vessel conditions (250 ${ }^{\circ} \mathrm{C}$, ca. 20 bar), and somewhat higher volumes (ca. $150 \mathrm{ml}$ ) under open vessel reflux conditions. In the much larger multi-mode instruments several liters can be processed under both open and closed vessel conditions. For both single- and multimode cavities continuous flow reactors are nowadays available that already allow the preparation of kilograms of materials using microwave technology.

\section{Chemistry Examples}

At the time of writing $c a .1000$ references on controlled microwave-assisted organic synthesis published since 2001 can be found in the literature. If one includes the many articles on MAOS describing work in domestic microwave ovens published since
1986 there are certainly more than 3000 references today documenting the many benefits of this enabling technology. A recent book with more than 1000 references summarized the many applications of controlled MAOS in organic chemistry [4d]. Almost any type of synthetic transformation known today has been evaluated under microwave conditions. These range from e.g. transition metal-catalyzed reactions, rearrangements and cycloadditions, glycosylations and peptide couplings, multicomponent reactions, free radical processes, to heterocyclic ring formations. Microwave synthesis has been successfully integrated with other technologies such as solid-phase synthesis, the use of solid-supported reagents or catalysts, fluorous reaction conditions and microreactor technology [4d]. The many advantages of this enabling technology have not only been exploited for organic synthesis [3][4] and in the context of medicinal chemistry/drug discovery [13], but have also penetrated fields such as polymer synthesis [14], material sciences [15], nanotechnology [16], and biochemical processes [17]. Literally every day another application is published in the literature, demonstrating the acceptance that this field has achieved in the 20 years since the first pioneering articles were published by Gedye and Giguere/Majetich [2].

\section{Summary and Outlook}

The steadily growing number of articles on MAOS appearing in the literature makes it obvious that almost any type of chemical transformation requiring heat can be carried out successfully under microwave conditions. This does not necessarily imply

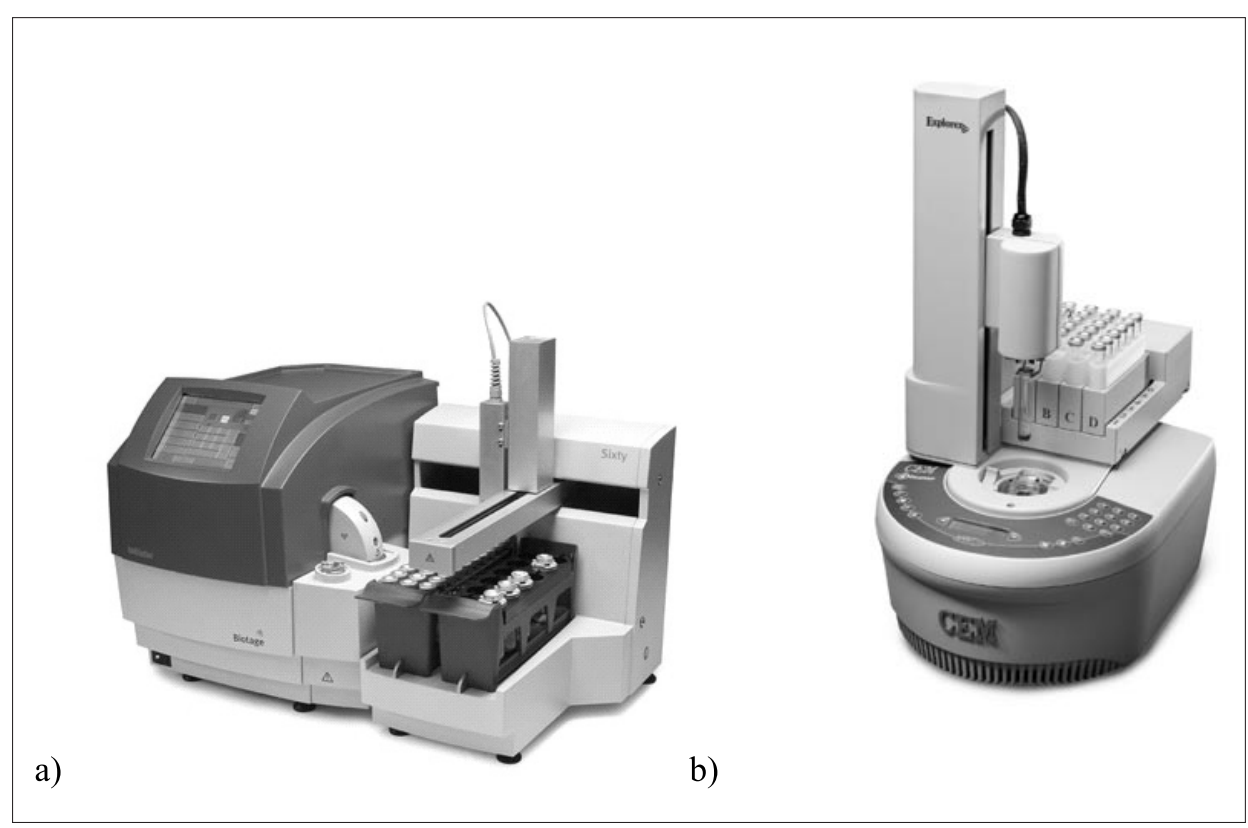

Fig. 3. Automated single-mode microwave reactors for MAOS (a: Initiator 60, Biotage AB; b: CEM Explorer, CEM Corporation). For a detailed survey of microwave equipment, see ref. [4d]. 
that dramatic rate-enhancements compared to a classical, thermal process will be observed in all cases, but the simple convenience of using microwave technology will make this non-classical heating method a standard tool in the laboratory within a few years. In the past, microwaves were often used only when all other options to perform a particular reaction failed, or when exceedingly long reaction times or high temperatures were required to complete a reaction. This practice is now slowly changing and due to the growing availability of microwave reactors in many laboratories, also routine synthetic transformations are now being carried out by microwave heating.

The benefits of controlled microwave heating, in particular in conjunction with using sealed vessel systems, are manifold.

- Most importantly, microwave processing frequently leads to dramatically reduced reaction times, higher yields, and cleaner reaction profiles. In many cases the observed rate-enhancements may be simply a consequence of the high reaction temperatures that can rapidly be obtained using this non-classical heating method, or may result from the involvement of so-called specific or non-thermal microwave effects.

- An additional benefit of this technology is that the choice of solvent for a given reaction is not governed by the boiling point (as in a conventional reflux setup) but rather by the dielectric properties of the reaction medium which can be easily tuned by $e . g$. addition of highly polar materials such as ionic liquids.

- The temperature/pressure monitoring mechanisms of modern microwave reactors allow for an excellent control of reaction parameters which generally leads to more reproducible reaction conditions.

- Because direct 'in core' heating of the medium occurs, the overall process is more energy efficient than classical oilbath heating.

- Microwave heating can be rapidly adapted to a parallel or automatic sequential processing format. In particular the latter technique allows for the rapid testing of new ideas and high-speed optimization of reaction conditions. The fact that a 'yes or no' answer for a particular chemical transformation can often be obtained within 5 to $10 \mathrm{~min}$ (as opposed to several hours in a conventional protocol), has contributed significantly to the acceptance of microwave chemistry both in industry and academia.

So why is not everybody using microwaves? One of the major drawbacks of this relatively new technology is equipment cost. While prices for dedicated microwave reactors for organic synthesis have come down considerably since their first introduction in the late 1990s, the current price range for microwave reactors is still many times higher than that of conventional heating equipment. As with any new technology, the current situation is bound to change over the next several years and less expensive equipment should become available. By then, microwave reactors will have truly become the 'Bunsen burners of the 21st century' and will be standard equipment in every chemical laboratory. In summary, MAOS has changed the world of organic chemistry, and it would be wise to embrace this new technology or be left lagging behind with conventional heating methodologies.

Received: March 24, 2006

[1] D. Adam, Nature 2003, 421, 571.

[2] a) R. Gedye, F. Smith, K. Westaway, H. Ali, L. Baldisera, L. Laberge, J. Rousell, Tetrahedron Lett. 1986, 27, 279; b) R.J. Giguere, T.L. Bray, S.M. Duncan, G. Majetich, Tetrahedron Lett. 1986, 27, 4945.

[3] For recent reviews, see: a) C.O. Kappe', Angew. Chem. Int. Ed. 2004, 43, 6250 and references cited therein; b) B.L. Hayes, Aldrichim. Acta 2004, 37, 66; c) M. Nüchter, B. Ondruschka, W. Bonrath, A. Gum, Green Chem. 2004, 6, 128; d) M. Nüchter, U. Müller, B. Ondruschka, A. Tied, W. Lautenschläger, Chem. Eng. Technol. 2003, 26, 1207; e) P. Lidström, J. Tierney, B. Wathey, J. Westman, Tetrahedron 2001, 57, 9225.

[4] a) 'Microwaves in Organic Synthesis', Ed. A. Loupy, Wiley-VCH, Weinheim, 2002; b) B.L. Hayes, 'Microwave Synthesis: Chemistry at the Speed of Light', CEM Publishing, Matthews, NC, 2002; c) 'Microwave-Assisted Organic Synthesis', Eds. P. Lidström, J.P. Tierney, Blackwell Publishing, Oxford, 2005; d) C.O. Kappe, A. Stadler, 'Microwaves in Organic and Medicinal Chemistry', Wiley-VCH, Weinheim, 2005; e) D. Bogdal, 'Microwave-Assisted Organic Synthesis', Elsevier, Amsterdam, 2005.

[5] a) D.R. Baghurst, D.M.P. Mingos, Chem. Soc. Rev. 1991, 20, 1; b) C. Gabriel, S. Gabriel, E.H. Grant, B.S. Halstead, D.M.P. Mingos, Chem. Soc. Rev. 1998, 27, 213.

[6] a) L. Perreux, A. Loupy, Tetrahedron 2001, 57, 9199; b) A. De La Hoz; A. DiazOrtiz; A. Moreno, Chem. Soc. Rev. 2005, 34, 164.

[7] B.L. Hayes, M.J. Collins, Jr., World Patent Appl., Publication No. WO 04002617, 2004.

[8] a) A. Loupy, A. Petit, J. Hamelin, F. Texier-Boullet, P. Jacquault, D. Mathé, Synthesis 1998, 1213; b) R.S. Varma, Green Chem. 1999, 1, 43; c) M. Kidawi, Pure Appl. Chem. 2001, 73, 147.

[9] A. Laporterie, J. Marquié, J. Dubac, in 'Microwaves in Organic Synthesis', Ed.
A. Loupy, Wiley-VCH, Weinheim, 2002 p. 219.

[10] S. Deshayes, M. Liagre, A. Loupy, J.-L. Luche, A. Petit, Tetrahedron 1999, 55, 10851.

[11] a) A.K. Bose, B.K. Banik, N. Lavlinskaia, M. Jayaraman, M.S. Manhas, Chemtech 1997, 27, 18; b) A.K. Bose, M.S. Manhas, S.N. Ganguly, A.H. Sharma, B.K. Banik, Synthesis 2002, 1578

[12] B.A. Roberts, C.R. Strauss, Acc. Chem. Res. 2005, 38, 653.

[13] For reviews, see: a) M. Larhed, A. Hallberg, Drug Discov. Today 2001, 6, 406; b) B. Wathey, J. Tierney, P. Lidström, J. Westman, Drug Discov. Today 2002, 7 , 373; c) F. Al-Obeidi, R.E. Austin, J.F. Okonya, D.R.S. Bond, Mini-Rev. Med. Chem. 2003, 3, 449; d) W.D. Shipe, S.E. Wolkenberg, C.W. Lindsley, Drug Discov. Today: Technol. 2005, 2, 155; e) C.O. Kappe, D. Dallinger, Nature Rev. Drug Discov. 2006, 5,55 .

[14] For reviews, see: a) D. Bogdal, P. Penczek, J. Pielichowski, A. Prociak, Adv. Pol. Sci. 2003, 163, 193; b) F. Wiesbrock, R. Hoogenboom, U.S. Schubert, Macromol. Rapid Commun. 2004, 25, 1739.

[15] For reviews, see: a) S. Barlow, S.R. Marder, Adv. Funct. Mater. 2003, 13, 517; b) Y.-J. Zhu, W.W. Wang, R.-J. Qi, X.-L. Hu, Angew. Chem. Int. Ed. 2004, 43, 1410.

[16] For a review, see: M. Tsuji, M. Hashimoto, Y. Nishizawa, M. Kubokawa, T. Tsuji, Chem. Eur. J. 2005, 11, 440.

[17] a) K. Orrling, P. Nilsson, M. Gullberg, M. Larhed, Chem. Commun. 2004, 790; b) H. Zhong, Y. Zhang, Z. Wen, L. Li, Nature Biotechn. 2004, 22, 1291; c) H. Zhong, S.L. Marcus, L. Li, J. Am. Soc. Mass Spectrom. 2005, 16, 471 . 